\title{
December 2014 Critical Care Case of the Month: Weak for Weeks
}

\author{
Bhupinder Natt $M D^{1}$ \\ Shadi Koleilat MD ${ }^{2}$ \\ Janet Campion $M D^{1}$ \\ ${ }^{1}$ Division of Pulmonary, Allergy, Critical care and Sleep Medicine \\ ${ }^{2}$ Department of Neurology \\ University of Arizona Medical Center \\ Tucson, AZ
}

\begin{abstract}
History of Present Illness
A 65 year old woman presents with weakness involving both upper and lower extremities that is intermittent over the last 3 months, but in the last 2 weeks she has also noticed increasing neck weakness, droopy eyelids and increased drooling. Prior to this she was able to walk without difficulty and ride a recumbent bike for 20 minutes, but now is having difficulty walking on her own. She denies fevers, weight loss, shortness of breath, chest pain, palpitations, LE edema, joint pain, rash, any recent or current GI/GU symptoms and no new medications.
\end{abstract}

Past Medical History, Social History, and Family History

The patient has a past history of hypertension, hyperlipidemia, diabetes mellitus Type II, GERD, obstructive sleep apnea (compliant with BiPAP), atrial fibrillation and hypothyroidism. She has a 40 pack-year history of tobacco use. Family history is noncontributory.

\section{Medications}

- Dabigatran 75mg BID

- Esomeprazole 20 mg BID

- Furosemide 30 mg BID

- Insulin glargine 50 Units BID and Lispro per sliding scale

- Levothyroxine $88 \mathrm{mcg}$ per day

- Losartan 50 mg QD,

- Pregabalin $75 \mathrm{mg}$ BID

- Rosuvastatin 40 mg per day

\section{Physical Examination}

Vital signs: Afebrile. Pulse 86, respiratory rate 20, PaO2 92\% on room air General: Awake, fully oriented, dysarthric speech.

HEENT: Non-icteric, ears, nares, oropharynx unremarkable; there is no neck LAD, elevated JVP or thyromegaly.

Respiratory: Normal breath sounds, no wheeze or rhonchi.

CVS: Irregularly irregular rhythm, no murmurs. Peripheral vascular exam normal. Abdomen: Obese, soft, non-tender with normal bowel sounds. No organomegaly appreciable.

Extremities: Trace pedal edema, normal muscle bulk and tone. 
CN: Ptosis bilaterally, no nystagmus, reactive pupils, extra-ocular muscles intact, sensation intact, weak cheek puff, symmetric palate excursion, normal tongue protrusion.

Motor: Neck flexion and extension 4-/5, bilateral pronator drift, no focal lower extremity weakness, no muscle atrophy, no tremors or fasciculations.

Sensation: Intact to light touch hands and feet.

Reflexes: 2+ and symmetric throughout.

Gait: Wide-based and slow, can only walk short distances before experiencing bilateral leg weakness.

Laboratory: Normal electrolytes, complete blood count, and liver function tests.

Creatinine mildly elevated at $2.1 \mathrm{mg} / \mathrm{dL}$.

\section{EKG}

Atrial Fibrillation

What is the most likely diagnosis?

1. Guillain-Barré syndrome (GBS)

2. Hypothyroidism

3. Lambert-Eaton myasthenic syndrome (LEMS)

4. Motor neuron disease (ALS)

5. Myasthenia gravis crisis 


\section{Correct! \\ 5. Myasthenia gravis crisis}

Myasthenia gravis (MG) is an autoimmune syndrome characterized by dysfunction of neuromuscular junction transmission due to autoantibody attack on the post-synaptic terminal. Patients often present with symptoms of fatigable weakness of bulbar, ocular and appendicular muscles. Weak cough (and difficulty handling secretions) and use of accessory breathing muscles are typical warning signs of imminent respiratory failure in MG.

MG can be difficult to distinguish from other causes of muscle weakness. Hypothyroidism typically presents as fatigue, constipation, cold intolerance and arthralgias/myalgias rather than muscle weakness. ALS typically presents with asymmetric limb weakness with hyperreflexia and spasticity, along with muscle atrophy and fasciculations; dysarthria and dysphagia may also be present. GBS presents as acute development of diffuse weakness and areflexia. LEMS is typically slowly progressive proximal (leg > arm) muscle weakness with autonomic dysfunction such as dry mouth, sluggish pupillary light reflex, and impotence. LEMS is unique as can see improvement in muscle strength with brief vigorous muscle activation.

What is the usual work up for this constellation of symptoms?

1. Autoantibodies

2. CT chest

3. EMG

4. $\mathrm{TSH}$

5. All of the above 


\section{Correct! \\ 5. All of the above}

TSH - approximately $30 \%$ of affected myasthenia gravis patients have a concomitant thyroid disease.

EMG - A very sensitive (97-99\%) test, but with low specificity (decremented response is also seen in Lambert-Eaton myasthenic syndromes, botulism toxin).

Autoantibodies - Autoantibodies against the postsynaptic acetylcholine receptor (AChRAbs) or against muscle-specific tyrosine kinase receptors (MuSK) occur in most patients. AChR-Abs come in 3 types: binding, blocking or modulating, with binding being the most sensitive (present in $80-90 \%$ of patient s with generalized disease.) Binding AChR-Abs rarely seen in other disorders: primary biliary cirrhosis, SLE, thymoma without myasthenia. MuSK antibodies should be tested when AChR antibodies are negative, particularly among patients with prominent bulbar muscle weakness.

CT of chest - Approximately $75 \%$ of affected patients have some sort of thymus abnormalities (hyperplasia, thymoma, etc); other associations include small cell cancer of the lung, breast cancer, Hodgkin lymphoma.

What are management options for myasthenia crisis?

1. Assessment of degree of weakness, bulbar dysfunction and respiratory status

2. Cholinesterase inhibitors

3. Incentive spirometry, assisted cough, sitting posture, frequent suctioning

4. Non-invasive ventilation in patients without hypercapnea

5. All of the above 


\section{Correct! \\ 5. All of the above}

Early initiation of immunomodulatory therapy and early use of BiPAP even in patients without hypercarbia can avert the need for mechanical ventilation, but the effectiveness of non-invasive ventilation declines once the patient has developed hypercapnea.

Initiation of non-invasive ventilation in MG patients with a $\mathrm{PaCO} 2<45 \mathrm{~mm} \mathrm{Hg}$ has been associated with reduced ICU and hospital lengths of stay and a decrease in pulmonary complications despite the presence of bulbar weakness. Facial weakness with poor oral seal and fluctuating degree of muscle weakness makes pulmonary function testing in MG patients unreliable.

Cholinesterase inhibitors such as oral pyridostigmine or parenteral neostigmine can be transiently discontinued to minimize respiratory secretions, but should always be restarted. Immunotherapy consists of either immunoglobulin (IVIG) or plasma exchange guided by clinician preference individualized for each patient. Caution should be exercised with high-dose steroids as one-third or one-half of patients will experience clinically significant weakness within days of starting treatment. Steroid-sparing immunosuppressant such as azathioprine or mycophenolate mofetil may be added to facilitate reduction of steroid dose.

What medications should be avoided in myasthenia gravis crisis?

1. Beta blockers

2. Calcium channel blockers

3. Ciprofloxacin

4. Morphine

5. All of the above 


\section{Correct! \\ 5. All of the above}

Myasthenic crisis may be precipitated by various factors, commonly respiratory infections, but certain medications such as beta blockers, calcium channel blockers, neuromuscular blocking agents, narcotics, aminoglycosides and fluoroquinolones have been known to interfere with neuromuscular junction transmission and should be avoided if possible. During intubation, if neuromuscular blocking agent is needed, it is preferable to use lower dose of non-depolarizing blocking agent $(0.5 \mathrm{mg} / \mathrm{kg}$ of rocuronium instead of the usual $1 \mathrm{mg} / \mathrm{kg}$ ) to prevent potential prolonged paralysis.

\section{What is cholinergic crisis?}

1. Excessive anticholinergic medication resulting in weakness

2. Hyperthermia

3. Psychosis

4. Pupillary constriction resulting in blindness

5. Urinary retention leading to bladder rupture 


\section{Correct! \\ 1. Excessive anticholinergic medication resulting in weakness}

Cholinergic crisis is a rare, but potentially major, side effect of excessive anticholinergic medication characterized by weakness. It may be difficult to distinguish this paradoxical weakness caused by the anticholinergic medication from the worsening myasthenia syndrome itself. Cholinergic crisis is rarely seen when the dosing limitation of pyridostigmine are followed: $<120 \mathrm{mg}$ every 3 hours OR $<960 \mathrm{mg}$ total daily dose. If these parameters are followed and the patient continues to have severe weakness, it is reasonable to assume that the MG is worsening.

\section{References}

1. Flower O, Bowles C, Wijdicks E, Weingart SD, Smith WS. Emergency neurological life support: acute non-traumatic weakness. Neurocrit Care. 2012; 17:S79-95. [CrossRef] [PubMed]

2. Kramer CL, Wijdicks EFM, Rabinstein AA. Acute Neuromuscular Disorders. Neurocritical Care Society 2013 Practice Update and Practice Exam. Available at: http://www.neurocriticalcare.org/sites/default/files/pdfs/13.Neuromusc.final.pdf (accessed 11/24/14). 